point is determined from the flow signal and controlled by the 'termination sensitivity'. As this is adjustable it seems hard to argue that the baby is in control of the inflation time, particularly without adequate monitoring. Our technique measures and shows the baby's spontaneous inspiratory time during inflation.

The use of this technique to display continuously the spontaneous respiratory pattern and compare it with the ventilator will enable the accurate detection of potentially harmful interactions between the baby and ventilator inflations - that is, spontaneous exhalation during ventilator inflation. At present this cannot be done and babies who appear to 'fight the ventilator' are often sedated or paralysed. This new technique should enable ventilators to be adjusted to reduce harmful interactions. We have shown that a regular sequence of ventilator inflations, at inflation and deflation times 'chosen' by the baby, can lead to phase-locking and more consistent synchrony. We hope to publish results of these early studies shortly.

It is clearly not possible to know how the ventilator and baby are interacting in the absence of any monitoring of the relevant signals. We believe that such monitoring is essential to improve the understanding of how babies interact with their ventilator, regardless of the mode of ventilation.

\section{Interleukin-8: a potent neutrophil chemotactic factor}

EDITOR,-Newborn infants, particularly those born prematurely, have an increased incidence of neutropenia. We reviewed retrospectively the results of an analysis of inflammatory mediators in the bronchoalveolar lavage fluid (BAL) of preterm infants with proved pulmonary infections in order to examine the recruitment of neutrophils into inflamed lung tissue in relation to the number of circulating neutrophils. We correlated the concentrations of interleukin-8 (IL-8), a potent neutrophil chemotactic factor, ${ }^{1}$ to those of neutrophils in the BAL of eight infants who presented with either normal (5) or decreased (3) absolute neutrophil count (ANC). In all the neonates IL-8 BALF concentrations were raised (mean (SD) 5.233 (1.001) $\mathrm{ng} / \mathrm{ml}$ ). However, neutrophil BAL concentrations appeared to be high (mean (range) $\left.1.5(0.9-1.8) \times 10^{6}\right)$ in infants with normal or increased ANC (mean (range) $4586(1512-10280) \mathrm{mm}^{3}$ ) whereas they were low (mean (range) $\left.0.01(0.0-0.03) \times 10^{6}\right)$ in those with decreased ANC values (mean (range) $473(373-655) \mathrm{mm}^{3}$ ). There was a significant association $\left(r_{\mathrm{s}}=0.78, \mathrm{p}<0.001\right)$ between IL-8 concentrations and the total number of lavage neutrophils in infants who presented with normal or increased ANC but not in those with low counts. These data suggest that adequate ANC values are essential for the recruitment of neutrophils into the lung. As the effects of neutrophils on bacterial removal depend on the number of neutrophils at the site of infection, treatments promptly applied to restore the number of circulating neutrophils could be useful in defending preterm infants from pulmonary infectious processes.

PAOLA PAPOFF PATRIZIA FIORUCCI CARLO OTTAVIANO GIOVANNI BUCCI Institute of Paediatrics, La Sapienza University of Rome, Viale $R$ Elena, 324 00161-Rome, Italy

1 Kunkel SL, Standiford T, Kasahara K, Strieter RM. Interleukin-8: the major neutrophil chemotactic factor in the lung. Exp Lung Res 1991; 17: 17-23.

\section{BOOK REVIEW}

The newborn infant: One brain for life. Edited by Claudine Amiel-Tison and Ann Stewart. (Pp 307, £69.95 hardback.) INSERM, 1994. ISBN 85598-571-4.

To deliver most women of their babies normally without cerebral injury to the fetus is the central aim of all perinatal units. The editors have sought contributions to the debate regarding the balance of risk around birth from neonatal paediatricians, obstetricians, anaesthetists, experimental psychologists and a psychoanalyst.

There is much useful material here, well presented, and with a refreshingly original approach. The emphasis is on the term infant and the process surrounding his or her maturation into a fetus competent to survive the birth process unscathed. The consideration of these events from several points of view was interesting and enjoyable reading. I particularly benefited from Professor AmielTison's elegant dissection of the underlying factors, with a characteristically useful section on assessing tone during the neurological examination of the infant. The section on metabolic/cardiovascular/endocrine adaptation around birth went over ground that is well trodden, but Professor Hugo Lagercrantz presented the material in a thorough, concise, and easily assimilated form.

A section on language acquisition, a topic that is rarely discussed, reminded me that the infant can classify syllables and differentiate early both his own language and his own mother's voice. Differences between pregnancies complicated by pre-eclampsia, intrauterine growth retardation, and the presence of another fetus were well reviewed. Some ex-cathedra statements sent me searching for confirmation, however. Can very frequent cardiotocography really prevent periventricular leucomalacia in pregnancies complicated by growth retardation?

Any strategy for reversing the upward trend in caesarean section deliveries is welcome, and several are suggested here. A decade of experience in Baudelaire Hospital, Paris is reported. The hospital delivers between 1000 and 2000 women every year and there was a reduction in the emergency caesarean section rate from $13 \%$ to $8 \%$ after a second opinion was made mandatory. This was accomplished without an apparent increase in the number of infants presenting with neurological symptoms after birth. Although this was an interesting approach, the result was not significant and the numbers were small. I was not sure that this material would have stood up to critical peer review. I mention this irritation because there seems to be an increasing tendency to use chapters in books to publish personal case series, which I would like to discourage.

Some of the contributions regarding preterm infants were brief to the point of being cryptic and could have been omitted. However, this would have meant the loss of an excellent review on the latest thoughts on the value of careful positioning to prevent muscle shortening in preterm infants. Peter Hope compared the value of alternative neurological investigations in a useful well referenced review. The last section, on medicolegal implications, was the most disappointing. The blurb on the jacket cover implied that the authors had grasped the nettle of this 'hot topic' but if you are hoping for a handy collection of material to assist you in clearing the backlog of expert witness reports piling up on your desk you will have to wait for another publication. The most frequent pregnancy complications which give rise to litigation if followed by handicap are ante-partum haemorrhage and failure to progress: no surprises here. The citations were scanty (mostly the author's own work) and the suggestion that the whole litigation crisis can be solved by a complete change in the insurance system is not going to affect the burgeoning problem in the United Kingdom.

Do not let the rather pretentious title or my small gripes put you off: for a while I admit alternative suggestions for the title prevented my concentrating on your behalf. I could not avoid mental speculation about where the emphasis should be put, and wondering what other organs (liver? heart?) could replace the brain. I hope it is clear from my review that I was glad I overcame this distraction.

J RENNIE 
techniques other than the lucid chapters of this book.

The central theme, respiratory failure in the newborn, is dealt with from a mainly physiological perspective. The aim is to provide core concepts, not recipes for clinical practice. This may sound somewhat arcane but the book is anything but dry. The editors have introduced a new device to enliven the text - individual comments by guest experts, at points throughout the book. Haven't you often wanted to argue with the author while reading a paper or textbook? The expert commentators do this for you. Some of the comments are based on personal observation. For instance, I very much like Os Reynolds's interjections to watch the patient if you wish to understand the concept of the time constant in ventilatory management. An epigram which struck a chord with me was the comment by Donald Null that 'the monitor works least well on the patients who need it the most'. Not all the comments are quite so imaginative. Some of the more erudite are backed up by reference to published evidence.

There are outstanding sections on the physiology of gas exchange and the pros and cons of physiological monitoring devices. A clearer description of the effects of ventilation/perfusion imbalance on gas exchange would be hard to find. Another well written and comprehensive section concerns surfactant, although much of this information is available elsewhere. One notable omission concerns the particular form of neonatal respiratory failure which poses difficulties to all who deal with newborn babies - not just those who work in neonatal intensive care units: failure to establish adequate breathing at birth. A book dealing with respiratory failure in the newborn really should tackle this problem, however difficult. It does, after all, provide the 'bread and butter' of neonatal paediatricians. In contrast, some of the sections which are included - for instance, the chapter on nitric oxide therapy - are likely to be outdated before the book reaches the library shelves.

All in all, this is a rewarding book, fun to dip into and a valuable resource for all involved in neonatal mechanical ventilation.

MICHAEL SILVERMAN Professor of Medicine, University of Leicester

Manual of neonatal emergency X-ray interpretation. $P$ W $D$ Meerstadt and C Gyll. (Pp 291, £18.95 paperback.) W B Saunders Company Ltd, 1994. ISBN 0-7020-1567-9.

The most enjoyable aspect of revising for medical examinations has been to pit my wits against those who have prepared practise questions in the form of data, slides, and clinical scenarios and then finding out immediately if I was correct. I felt a similar enjoyment reading this book, which aims to teach junior doctors working in a neonatal unit to assess critically plain radiographs they are likely to encounter. Disorders likely to be encountered as emergencies are illustrated with a systematic text accompanying each radiograph. The invariable technical comments, although useful, were excessive and repetitive when reading the book from cover to cover.

The different sections take the reader from the normal through varying degrees of pathology, most of the clinical conditions being illustrated by several different radiographs. The final section details the positioning of clinical equipment and the traps that the unwary may fall into by misinterpreting artefacts.

The interpretation of neonatal films is often extremely difficult without a clinical history; such brief histories would have added to the book, particularly in cases where identical radiographic appearances may be caused by a number of conditions, the management of the baby depending on a combination of history and $x$-ray appearances.

Many of the films are accompanied by suggestions of further follow up films from different views to confirm the diagnosis. My main criticism is that in several of these, the diagnosis is obvious with all the information provided on the standard view, so further radiology is unnecessary.

The book contains a number of high quality $x$-ray images and will fill a gap in the market as a user-friendly manual for those working on neonatal intensive care units. It will also be useful to junior paediatricians about to sit examinations, and nurses aiming to become advanced practitioners.

AMANDA OGILVY-STUART Clinical Lecturer in Paediatrics, fohn Radcliffe Hospital, Oxford

Baillières Clinical Paediatrics. Pulmonary Problems in the Perinatal Period and their Sequelae. Edited by V Y H Yu. (Pp 245; £30 hardback.) Harcourt Brace, 1995. ISBN 0-7020-1934-8.

It is not an easy task to produce a comprehensive text which attempts to give adequate coverage to both common neonatal respiratory conditions and useful information on rare respiratory diagnoses, all in under 250 pages. To a large degree this book, produced as part of Baillière's Clinical Paediatrics series, achieves this successfully with its list of eminent international contributors who have written readable, comprehensive chapters with an extensive bibliography after each.

Some of the chapters inevitably reflect the individual's personal view, for example the use of shouldered tubes during neonatal resuscitation. Other parts are disappointingly brief, for example in the discussion of the role and mechanism of the increasingly used nasal continuous positive airway pressure flow driver system employing the coander effect. There is also some variation of language and style which reflects the varied international origins of the contributors.

As there are many publications, large and small, on pulmonary problems in the perinatal period, I was keen to see what this volume offered that was new or different. The cover of Baillière's clinical paediatrics suggested that the contents of the publication would be based around international practice and research. I was however, a little disappointed to find that the majority of the chapters avoided discussing any differences in international practice nor was there much presentation of research from the interesting and controversial areas of clinical practice, tending towards the citation of well accepted facts and long published data. For example, there is discussion of the fact that antenatal steroids are useful for the prevention of respiratory distress syndrome, but the authors have rather skated over the more controversial use of thyroid releasing hormone, with a mention in just one paragraph and no discussion of any published data on its efficacy or mechanism of action.

There is a generally useful chapter on the assessment of pulmonary function, although some of the equations left me confused. The final chapter concentrates on new technology with sections on a range of treatments increasingly employed in larger neonatal units, such as high frequency oscillation and nitric oxide, and includes two pages given over to the futuristic modalities of total or partial liquid ventilation.

Overall this volume covers a wide range of pulmonary problems and treatments with the major emphasis rightly being given to the common problems, while other chapters provide one or two brief paragraphs on each of many congenital thoracic and pulmonary abnormalities which appear from time to time. The detail provided is at a level that falls between a definitive text and that found in a variety of neonatal handbooks, and would provide a good readable basis for anyone wanting to update their knowledge in the ever changing area of pulmonary management of the newborn infant.

\section{ANTHONY J B EMMERSON}

Consultant paediatrician

\section{Correction}

In a letter to the Editor entitled Interleukin-8: a potent neutrophil chemotactic factor (Arch Dis Child, 1995; 73: F54) the name of the third author, Carla Ottaviano, was erroneously printed as Carlo Ottaviano. We apologise to Dr Ottaviano for this mistake. 